\title{
THE
}

\section{The Influence of Diffusion on Surface Reaction Kinetics}

David L. Freeman

University of Rhode Island, dfreeman@uri.edu

Jimmie D. Doll

Follow this and additional works at: https://digitalcommons.uri.edu/chm_facpubs

Terms of Use

All rights reserved under copyright.

\section{Citation/Publisher Attribution}

Freeman, D. L. \& Doll, J. D. (1983). The Influence of Diffusion on Surface Reaction Kinetics. J. Chem.

Phys., 78, 6002-6009. doi: 10.1063/1.444616

Available at: http://dx.doi.org/10.1063/1.444616

This Article is brought to you for free and open access by the Chemistry at DigitalCommons@URI. It has been accepted for inclusion in Chemistry Faculty Publications by an authorized administrator of DigitalCommons@URI. For more information, please contact digitalcommons-group@uri.edu. 


\section{AIP Chemicalit Prysics}

\section{The influence of diffusion on surface reaction kinetics}

David L. Freeman and J. D. Doll

Citation: J. Chem. Phys. 78, 6002 (1983); doi: 10.1063/1.444616

View online: http://dx.doi.org/10.1063/1.444616

View Table of Contents: http://jcp.aip.org/resource/1/JCPSA6/v78/i10

Published by the American Institute of Physics.

Additional information on J. Chem. Phys.

Journal Homepage: http://jcp.aip.org/

Journal Information: http://jcp.aip.org/about/about_the_journal

Top downloads: http://jcp.aip.org/features/most_downloaded

Information for Authors: http://jcp.aip.org/authors

\section{ADVERTISEMENT}

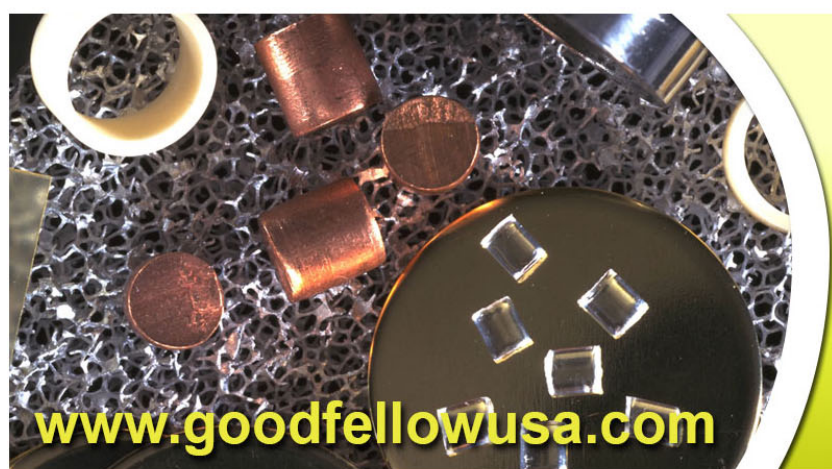




\title{
The influence of diffusion on surface reaction kinetics
}

\author{
David L. Freemana) and Jimmie D. Doll \\ University of California, Los Alamos National Laboratory, Chemistry Division, Los Alamos, New Mexico 87545 \\ (Received 16 December 1982; accepted 10 February 1983)

\begin{abstract}
kinetics. It is shown that a pure two-dimensional model of surface reactions yields no steady state rate constant. By incorporation of adsorption and desorption processes the deficiencies in the two-dimensional results are eliminated. Expressions are derived for diffusion-controlled and diffusion-influenced rate constants for surface reactions. Expressions are also derived for the activation energies of these surface reactions. It is shown that the activation energy for diffusion-controlled reactions will approximately be given by the activation energy for surface diffusion. Bounding expressions are developed for the activation energy for diffusion-influenced reactions. Comparisons are made between Langmuir-Hinshelwood and Eley-Rideal mechanisms, and it is found that Langmuir-Hinshelwood mechanisms should be more important than Eley-Rideal processes for many surface reactions.
\end{abstract} \\ An analysis is given of diffusion-influenced surface reactions using models similar to those used in solution
}

\section{INTRODUCTION}

It has long been recognized that diffusion is an important step in the kinetics of rapid reactions in solution. In the limit of very rapid reactions the diffusion step may be rate limiting and such reactions are termed diffusion controlled. Diffusion-controlled reactions in solution have been given an extensive theoretical treatment and an excellent review of the subject has been given by $\mathrm{Lin}, \mathrm{Li}$, and Eyring. ${ }^{1}$

For heterogeneous reactions occurring at the surface of solids it is manifest that diffusion is an important step for a variety of reactions. In analogy with solution kinetics many very rapid surface reactions can be expected to be diffusion controlled. For example when a dissociatively adsorbed diatomic molecule desorbs from the surface of a crystal it can be expected that the recombination of the atoms will occur very rapidly when the atoms reach a close critical distance. The rate limiting step for such a recombination reaction may prove to be the rate at which the atoms diffuse together on the surface.

For diffusion-controlled reactions in solution, information about the rates can be extracted from a knowledge of the diffusion constants of the reactants. ${ }^{1}$ Recently, diffusion constants associated with the migration of adsorbates on crystal surfaces have become available both theoretically ${ }^{2-5}$ and experimentally. ${ }^{6}$ As a consesequence, it is timely to determine expressions for rate constants in terms of diffusion constants for those cases where the influence of surface diffusion is large.

In the present work we analyze and develop expressions for the rate constants of diffusion-influenced surface reactions. Our treatment is based upon the Smoluchowski ${ }^{7,8}$-Collins-Kimball ${ }^{9}$ approach to solution kinetics and includes features used in theories of thin film nucleation. ${ }^{10}$ In a mechanistic sense we concentrate on those reactions which obey Langmuir-Hinshelwood kinetics ${ }^{11}$ between surface adsorbed species $s-A$ and

\footnotetext{
${ }^{a)}$ Visiting staff member at Los Alamos National Laboratory. Permanent address: Department of Chemistry, The Univeristy of Rhode Island, Kingston, Rhode Island 02881.
}

$$
\begin{aligned}
& s-B \text { to produce gas-phase products } A B_{(8)} \\
& s-A+s-B \frac{\stackrel{k}{\frac{k}{k^{\prime}}} 2 s+A B_{(B)},}{}
\end{aligned}
$$

as opposed to the classical Eley-Rideal mechanism ${ }^{11}$

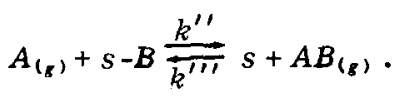

Our development will allow the construction of expressions to compare the relative rates of mechanisms (1) and (2).

The contents and organization of the remainder of this paper are as follows: In Sec. II we briefly review the treatment of diffusion-influenced reactions in solution. This review establishes the notation and methodology used in the remainder of the paper. In Sec. III we develop the formalism to study the influence of diffusion on surface reactions. We begin Sec. III by introducing a two-dimensional model for surface reactions. Although this model will be found to be unphysical the derived expressions will be useful in evaluating limits of the results discussed subsequently. We follow by developing a model which includes an adsorption and desorption mechanism. Within this model we develop expressions for reaction rate constants and analyze the expected behavior of the activation energy for diffusion-influenced surface reactions. We also develop an expression for the relative rates of Eley-Rideal to Langmuir-Hinshelwood processes. In Sec. IV we discuss the nature of the approximations used in our treatment and summarize our conclusions.

\section{REVIEW OF DIFFUSION-INFLUENCED REACTIONS IN SOLUTION}

Although diffusion-influenced reactions in solution have been treated extensively elsewhere ${ }^{1}$ the main body of the remainder of this paper will be clarified by briefly reviewing the treatment of Smoluchowski ${ }^{7,8}-$ Collins and Kimball. ${ }^{9}$ This review will also allow us to establish our methodology and notation.

We consider a solution whose initial state consists of reactant $A$ and $B$ molecules distributed uniformly. The 
molecules diffuse, and a reaction is allowed to occur whenever an $A$ and $B$ molecule come within a critical distance $R_{A}$ of each other. If $A$ and $B$ molecules a distance $R_{A}$ apart react with unit probability the reaction is said to be diffusion controlled. Otherwise, the reaction is said to be diffusion influenced.

It is useful to imagine an $A$ molecule as fixed and the $B$ molecules as diffusing towards the $A$ molecule. If we let $W_{S}(\mathbf{r}, t)$ be the probability that a $B$ molecule is at coordinate $\mathbf{r}$ at time $t$ relative to an unreacted $A$ molecule, then it can be shown ${ }^{8}$ that $W_{s}(r, t)$ satisfies the diffusion equation

$$
\frac{\partial W_{S}}{\partial t}=D \nabla^{2} W_{S},
$$

where $D$ is the sum of the diffusion constants for molecules $A$ and $B$; i.e.,

$$
D=D_{A}+D_{B} \text {. }
$$

Equation (4) will be valid whenever the motion of diffusing $A$ and $B$ molecules is uncorrelated. The function $W_{S}(\mathrm{r}, t)$ can also be interpreted as the concentration of $B$ molecules. The subtleties associated with these interpretations has been discussed in detail by Collins and Kimball. 9 In this work we will be concerned with the solutions to Eq. (3) at steady state; i.e., when

$$
\frac{\partial W_{s}(r, t)}{\partial t}=0 \text {. }
$$

Consequently, we seek solutions to Laplace's equation

$$
\nabla^{2} W_{\mathrm{ss}}=0 \text {, }
$$

where the subscript SS in Eq. (6) denotes steady state. For spherical reactants like atomic species Eq. (6) is best solved in spherical polar coordinates so that

$$
\frac{d^{2} W_{8 B}}{d r^{2}}+\frac{2}{r} \frac{d W_{8 \beta}}{d r}=0 \text {. }
$$

The boundary conditions imposed on Eq. (7) are the radiation boundary conditions of Collins and Kimball. ${ }^{9}$ At the critical reaction radius $R_{A}$, we set

$$
4 \pi R_{\mathrm{A}}^{2} D\left(\frac{d W_{g 8}}{d r}\right)_{r=R_{A}}=k_{\odot \mathrm{Qq}} W_{\mathrm{gg}}\left(R_{\mathrm{A}}\right),
$$

where $k_{\text {oq }}$ is the reaction rate constant once the reactants are at the critical radius. When $k_{\text {eq }}=0$ we have no reaction possible and obtain the pure reflection boundary condition

$$
\left(\frac{d W_{\text {Sg }}}{d r}\right)_{r=R_{A}}=0
$$

and when $k_{\text {ou }}$ is infinite we obtain the pure absorption boundary condition

$$
W_{\mathrm{sg}}\left(R_{\mathrm{A}}\right)=0 \text {. }
$$

The boundary condition given in Eq. (10) was used by Smoluchow $\mathrm{Ski}^{7,8}$ and is appropriate when the reaction is diffusion controlled. In this work we will find solutions subject both to boundary conditions (8) and (10) since both cases are physically important.

As a second boundary condition we surround our $A$ molecule with a fictitious outer boundary at $R_{B}$ and set

$$
W_{88}\left(R_{B}\right)=C_{0},
$$

where $C_{0}$ is the initial concentration of $B$ molecules. The boundary condition expressed in Eq. (11) is equivalent to finding steady-state solutions from an initial uniform concentration of $C_{0}$. The physical solution will be found in the limit that $R_{a}$ becomes infinite.

We begin by finding the solution to Eq. (7) using the absorption boundary condition given in Eq. (10). The solution found by standard techniques is

$$
W_{\mathrm{ss}}(r)=C_{0} \frac{\left(1-R_{A} / r\right)}{\left(1-R_{A} / R_{B}\right)}
$$

In the limit that $R_{B}$ becomes infinite we obtain

$$
\begin{aligned}
W_{\mathrm{BS}_{0}}(r) & =\lim _{R_{B} \rightarrow \infty} W_{88}(r), \\
& =C_{0}\left(1-\frac{R_{A}}{r}\right) .
\end{aligned}
$$

The diffusion-controlled rate constant for the reaction can be obtained from the defining relation

$$
\begin{aligned}
k_{3 D} & =\frac{4 \pi R_{A}^{2} D}{C_{0}}\left(\frac{d W_{\mathrm{SQ}}}{d r}\right)_{r=R_{\mathrm{A}}}, \\
& =4 \pi R_{\mathrm{A}} D .
\end{aligned}
$$

The expression for the diffusion-controlled rate constant given in Eq. (16) can be found in many textbooks. ${ }^{12}$

If the reaction is diffusion-influenced rather than diffusion-controlled solutions to Eq. (7) must be found using the boundary condition of Eq. (8). A solution by standard methods gives

$$
\begin{aligned}
W_{\mathrm{sB}_{1}}(r)= & C_{0}\left[1+\frac{k_{\text {ee }}}{k_{\mathrm{eq}} R_{B}\left(\frac{1}{R_{A}}-\frac{1}{R_{B}}\right)+4 \pi D R_{B}}\right. \\
& \left.-\frac{k_{\mathrm{eq}} / r}{k_{\text {eq }}\left(\frac{1}{R_{A}}-\frac{1}{R_{B}}\right)+4 \pi D}\right] .
\end{aligned}
$$

In the limit that $R_{B}$ becomes infinite we obtain

$$
\begin{aligned}
W_{\mathrm{ss}_{1 \infty}}(r) & \equiv \lim _{R_{B} \rightarrow \infty} W_{\mathrm{si}_{1}}(r) \\
& =C_{0}\left(\frac{1-k_{\mathrm{eQ}} R_{A} / r}{k_{\mathrm{eq}}+4 \pi D R_{A}}\right) .
\end{aligned}
$$

The diffusion-influenced rate constant is given by

$$
\begin{aligned}
k_{3 D 1} & =\frac{4 \pi R_{A}^{2} D\left(\frac{d W_{88_{1 \infty}}}{d r}\right)_{r \sim R_{A}}}{C_{0}}, \\
& =\frac{k_{3 D} k_{\text {ea }}}{k_{3 D}+k_{\text {eq }}} .
\end{aligned}
$$

An expression analogous to Eq. (21) was recently given by Szabo ef al. ${ }^{13}$ It is clear that the rate constant as sociated with the radiation boundary conditions of Eq. (8) can be obtained from the absorption boundary condition solution and the application of Eq. (21). When $k_{\text {eq }}$ is very large as in diffusion controlled processes

$$
\lim _{k_{\infty \rightarrow \infty} \rightarrow \infty} k_{3 D 1}=k_{3 D} \text {. }
$$

In general, 


$$
k_{3 D_{1}} \leq k_{3 D}
$$

so that an evaluation of the diffusion-controlled rate constant from Eq. (16) will give an upper bound to the true rate constant.

We can intuit Eq. (21) from simple time sequence considerations. For bimolecular processes like those we consider here the half-life of reactants for any step is given by

$$
t_{(1 / 2)_{i}}=\frac{1}{C_{0} k_{i}},
$$

where $k_{i}$ is the rate constant associated with step $i$. In the systems we consider the half-life of a reactant is equal to the half-life of the diffusion step plus the halflife of the reaction step, $i_{0} e_{\text {. , }}$

$$
t_{(1 / 2) 3 D 1}=t_{(1 / 2) 3 D}+t_{(1 / 2) \text { eq }},
$$

or

$$
\frac{1}{k_{3 D 1}}=\frac{1}{k_{3 D}}+\frac{1}{k_{O Q}}
$$

which is equivalent to Eq. (21).

\section{DIFFUSION-INFLUENCED REACTIONS ON SURFACES}

With our review of diffusion-influenced reactions in solution complete we are in a position to analyze the corresponding problem for surface reactions. Before beginning the analysis some differences between the surface and solution systems are useful to identify. In solution kinetics the diffusion is three-dimensional, whereas in surface chemistry the diffusion can be imagined to be quasi-two-dimensional. The difference in dimensionality will lead to some mathematical difficulties. Another important difference between surface and solution kinetics arises from the heterogeneous environment of a surface reaction. For a surface reaction the presence of reactants in the gas-phase results in an inflow and outflow of material via adsorption and desorption. Of course, no such adsorption or desorption events occur in solution reactions.

\section{A. A two-dimensional model}

Although adsorption and desorption can be expected to be important we begin our analysis by examining surface diffusion-influenced reactions in the absence of any mechanism which allows material to flow into or out of our system. Although we shall find the resulting solution to be unphysical we present the results for comparison with limiting forms of more physical models to be derived subsequently.

In polar coordinates the two-dimensional steady-state diffusion equation is

$$
\frac{d^{2} W_{2 D}}{d r^{2}}+\frac{1}{r} \frac{d W_{2 D}}{d r}=0
$$

If we apply absorbing boundary conditions to Eq. (27),

$$
\begin{aligned}
& W_{2 D}\left(R_{A}\right)=0, \\
& W_{2 D}\left(R_{B}\right)=C_{0},
\end{aligned}
$$

the solution to Eq. (27) is

$$
W_{2 D}(r)=\frac{C_{0} \ln \frac{r}{R_{A}}}{\ln \frac{R_{B}}{R_{A}}} .
$$

In the limit that $R_{B}$ becomes infinite $W_{2 D}(r)$ vanishes for all $r$. In two dimensions the effects of the absorbing boundary conditions are very long range and no finite steady-state concentration can be attained. Similar conclusions concerning two-dimensional behavior have been given by Emeis and Fehder. ${ }^{14}$ By implication no steady-state rate constant can be defined in two dimensions. To see this we define

$$
\begin{aligned}
k_{2 D} & =\frac{2 \pi R_{A} D}{C_{0}}\left(\frac{d W_{2 p}}{d r}\right)_{r=R_{A}}, \\
& =\frac{2 \pi D}{\ln \frac{R_{B}}{R_{A}}} .
\end{aligned}
$$

It is clear that $k_{2 D}$ also vanishes as $R_{B}$ becomes infinite.

If radiation boundary conditions rather than absorbing boundary conditions are used to solve Eq. (27) we write

$$
2 \pi R_{A} D\left(\frac{d W_{2 D}}{d r}\right)_{r=R_{A}}=k_{\theta Q} W_{2 D}\left(R_{A}\right) .
$$

It is easy to show that the resulting rate constant is given by

$$
k_{2 D 1}=\frac{k_{2 D} k_{\theta Q}}{k_{2 D}+k_{\text {eq }}}
$$

in complete analogy with Eq. (21). Because $k_{2 p}$ vanishes as $R_{B}$ becomes infinite it is clear that $k_{2 D 1}$ also vanishes.

The origin of the vanishing rate constant in the twodimensional model is the neglect of adsorption and desorption processes. It is not the result of unphysically confining material to be purely two-dimensional. We will introduce an adsorption-desorption mechanism in the next section. Before leaving this section we consider the behavior of diffusing material confined to a slab of thickness $l$. We consider an absorber of radius $R_{A}$ placed at the origin of our coordinate system. Concentric with the absorber we place an outer sphere of radius $R_{B}$. We seek solutions to the three-dimensional steady-state diffusion equation in cylindrical coordinates

$$
\frac{\partial^{2} W_{2 D Z}}{\partial r^{2}}+\frac{1}{r} \frac{\partial W_{2 D Z}}{\partial r}+\frac{\partial^{2} W_{2 D Z}}{\partial z^{2}}=0
$$

subject to the boundary conditions

$$
\begin{aligned}
& W_{2 D Z}\left(R_{A}, z\right)=0, \\
& W_{2 D Z}\left(R_{B}, z\right)=C_{0},
\end{aligned}
$$

and

$$
\left(\frac{\partial W_{2 D z}}{\partial z}\right)_{z=1 / 2}=0
$$

The boundary condition expressed in Eq. (38) imposes perfect reflection at $z= \pm(l / 2)$ and confines material to flow in a slab of thickness $l$. For small $l$ we have diffusion in a thin film which is more physical than our 
original two-dimensional model.

We can immediately write that

$$
\lim _{R_{B} \rightarrow \infty} \lim _{r \rightarrow R_{B}} W_{2 D Z}(r, z)=W_{2 D}(r) .
$$

Equation (39) follows from the fact that $W_{2 D}(r)$ satisfies Eq. (35) along with the boundary conditions [Eqs. (36)(38) ] at the limit. The rate constant can be written

$$
k_{2 D z}=\frac{2 \pi R D}{C_{0}}\left(\frac{d W_{2 D Z}}{d r}\right)_{r=R},
$$

where the derivative is evaluated at any radius $R, R$ $\gg l$, at steady state. If we choose $R$ to be $R_{B}$, we see that

$$
\lim _{R_{B} \rightarrow \infty} k_{2 D Z}=0 \text {. }
$$

Consequently, no finite steady-state rate constant exists for a thin film in the absence of adsorption and desorption processes. The difficulty with the solution to Eq. (27) does not arise from unphysically considering matter to be two-dimensional. We have checked these conclusions numerically by Monte-Carlo solution of Eq. (35) ${ }^{15,16}$

\section{B. Inclusion of absorption and desorption processes}

The loss and capture of reactant molecules from the gas phase can be investigated from the modified twodimensional diffusion equation

$$
\frac{\partial W}{\partial t}=D \nabla^{2} W+J-\frac{W}{\tau},
$$

where $\tau$ is the lifetime of an absorbed reactant to desorption and $J$ is the amount of reactant per unit time per unit area flowing onto the surface. This same equation has been used to study thin film nucleation processes ${ }^{10}$ although the boundary conditions appropriate for thin film nucleation are somewhat different than those we use for reaction kinetics. At steady state we solve

$$
D \tau\left(\frac{d^{2} W_{D}}{d r^{2}}+\frac{1}{r} \frac{d W_{D}}{d r}\right)-W_{D}+J \tau=0 .
$$

Only two parameters occur in Eq. (43); $D \tau$ and $J \tau$. The flow of reactant onto the surface represented by $J$ can either arise from an external source or from an equilibrium vapor phase. To understand this equivalence we follow Langmuir ${ }^{17}$ and assume that the desorption rate is proportional to $W_{D}$ and the adsorption rate is proportional to $W_{0}-W_{D}$ where $W_{0}$ is the concentration on the surface at full coverage. Then

$$
\frac{\partial W_{D}}{\partial t}=D \nabla^{2} W_{D}-\frac{W_{D}}{\tau_{d}}+\frac{W_{0}-W_{D}}{\tau_{a}},
$$

where $\tau_{d}$ is the desorption lifetime and $\tau_{a}$ is the lifetime of atoms in the gas phase. If we identify $\tau^{-1}$ with $\left(\tau_{a}^{-1}\right.$ $\left.+\tau_{d}^{-1}\right)$ and $J$ with $\left(W_{0} / \tau_{a}\right)$ then we see Eq. (44) is equivalent to Eq. (42).

We now solve Eq. (43) with absorbing boundary conditions corresponding to diffusion-controlled reactions. We use the boundary conditions

$$
W_{D}\left(R_{A}\right)=0 \text {, }
$$

$$
W_{D}\left(R_{B}\right)=C_{0} .
$$

As before, the physical solution to Eq. (43) will occur for infinite $R_{B}$. Using elementary methods Eq. (43) can be solved to give

$$
W_{D}(r)=J \tau\left[1+A K_{0}(\gamma r)+B I_{0}(\gamma r)\right] \text {, }
$$

where $I_{n}(x)$ and $K_{n}(x)$ are modified Bessel functions of the first and second kind of order $n$,

$\gamma=(D \tau)^{-1 / 2}$,

$A=-\frac{1}{K_{0}\left(\gamma R_{A}\right)}\left\{1+\frac{I_{0}\left(\gamma R_{A}\right) K_{0}\left(\gamma R_{A}\right)\left[\frac{C_{0}}{J \tau}-1+\frac{K_{0}\left(\gamma R_{B}\right)}{K_{0}\left(\gamma R_{A}\right)}\right]}{K_{0}\left(\gamma R_{A}\right) I_{0}\left(\gamma R_{B}\right)-I_{0}\left(\gamma R_{A}\right) K_{0}\left(\gamma R_{B}\right)}\right\}$,

and

$B=\frac{K_{0}\left(\gamma R_{A}\right)}{K_{0}\left(\gamma R_{A}\right) I_{0}\left(\gamma R_{B}\right)-I_{0}\left(\gamma R_{A}\right) K_{0}\left(\gamma R_{B}\right)}\left[\frac{C_{0}}{J \tau}-1+\frac{K_{0}\left(\gamma R_{B}\right)}{K_{0}\left(\gamma R_{A}\right)}\right]$.

The parameter $\gamma$, defined by $\mathrm{Eq} .(48)$ is the inverse of half the average distance traveled by a reactant molecule in the time before desorption.

Before we let $R_{B}$ become infinite it is of interest to determine the behavior of Eq. (47) as $\gamma$ approaches zero. This limit corresponds to long absorption lifetimes and large diffusion constants. To determine the limits we use well known expressions ${ }^{18}$ for Bessel functions. From Eq. (49),

$$
\lim _{\gamma \rightarrow 0} A=-\frac{C_{0}}{J \tau \ln \frac{R_{B}}{R_{A}}}
$$

and from Eq. (50)

$$
\lim _{r \rightarrow 0} B=-\left(1+\frac{C_{0}}{J \tau \ln \frac{R_{B}}{R_{A}}}\right) \text {. }
$$

Then

$$
\begin{aligned}
\lim _{r \rightarrow 0} W_{D}(r) & =J \tau\left[1+\frac{C_{0} \ln \gamma r}{J \tau \ln \frac{R_{B}}{R_{A}}}-1-\frac{C_{0} \ln \gamma R_{A}}{J \tau \ln \frac{R_{B}}{R_{A}}}\right], \\
& =\frac{C_{0} \ln \frac{r}{R_{A}}}{\ln \frac{R_{B}}{R_{A}}}, \\
& =W_{2 D}(r) .
\end{aligned}
$$

We see that $W_{D}(r)$ is equivalent to $W_{2 D}(r)$ for small $\gamma$ and finite $R_{B}$. It is of interest that $W_{D}(r)$ becomes independent of $J$ as $\gamma$ becomes small.

The physical solution occurs for infinite $R_{B}$. Again using well known properties of Bessel functions it is elementary to show that

$$
\begin{aligned}
\lim _{R_{B} \rightarrow \infty} W_{D}(r) & \equiv W_{\infty}(r), \\
& =J \tau\left[1-\frac{K_{0}(\gamma r)}{K_{0}\left(\gamma R_{A}\right)}\right] .
\end{aligned}
$$

By incorporating an adsorption and a desorption process into the two-dimensional diffusion equation we find 


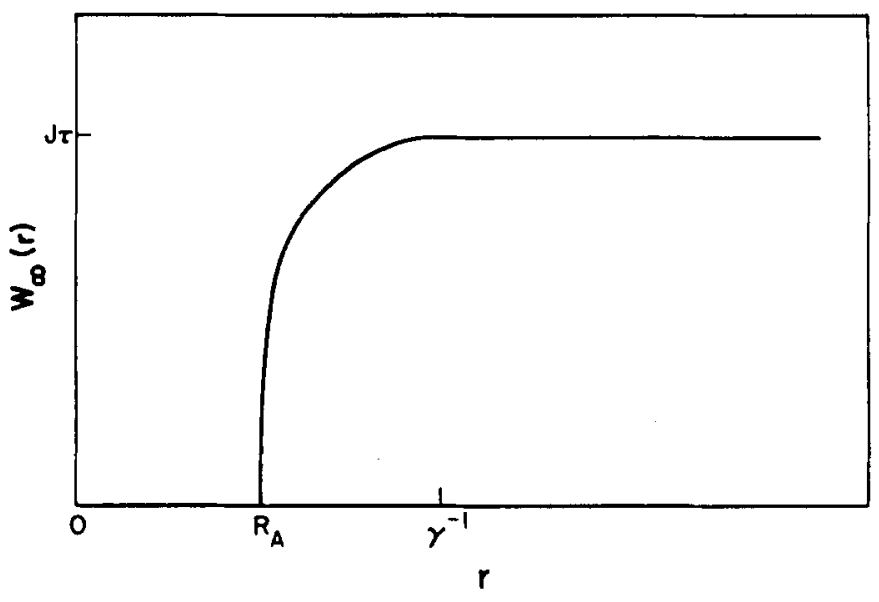

FIG. 1. Graph of the concentration of reactant molecules as a function of the distance from the absorber [Eq. (57)].

a finite and well behaved solution for $W_{D}(r)$ at infinite $R_{B}$. Another interesting limit is

$$
\begin{aligned}
\lim _{r \rightarrow \infty} W_{\infty}(r) & =J \boldsymbol{\tau}, \\
& \equiv C_{\infty},
\end{aligned}
$$

which is the steady-state concentration far from the adsorber. To gain further insight into the behavior of $W_{\infty}(r)$ we consider the graph of $W_{\infty}(r)$ shown in Fig. 1 . We find in the circular region of radius $\gamma^{-1}$ about the reacting $A$ molecule a depletion of $B$ molecules. For distances beyond $\gamma^{-1}$ from the $A$ molecule the concentration of $B$ rapidly approaches $C_{\infty}$, a constant.

Because of the flux of reactant molecules from the vapor phase the reaction rate in this model contains two terms. One term is a consequence of the diffusion process and is similar to the rate expressions we considered previously. In addition there is a term corresponding to the reaction of adsorbed molecules with reactant molecules from the gas phase. Incorporating both terms we obtain the rate expression

$$
F=F_{A}+F_{D},
$$

where

$$
F_{A}=\pi R_{A}^{2} J
$$

is the direct adsorption rate, and

$$
F_{D}=2 \pi R_{A} D\left(\frac{d W_{\infty}}{d r}\right)_{r=R_{A}}
$$

is the diffusion rate. We will discuss the importance of $F_{A}$ in Sec. III C when we compare the Eley-Rideal and Langmuir-Hinshelwood mechanisms mentioned in the Introduction. Presently, we evaluate Eq. (62) from Eq. (57) to obtain

$$
F_{D}=2 \pi R_{A} D J \tau \gamma \frac{K_{1}\left(\gamma R_{A}\right)}{K_{0}\left(\gamma R_{A}\right)}
$$

If we let

$$
\lambda=\frac{\gamma K_{1}\left(\gamma R_{A}\right)}{K_{0}\left(\gamma R_{A}\right)}
$$

Eq. (63) becomes

$$
F_{D}=2 \pi R_{A} D \lambda C_{\infty} \text {. }
$$

By definition the diffusion-controlled rate constant is

$$
k_{D}=\frac{F_{D}}{C_{\infty}}
$$

so that

$$
k_{D}=2 \pi R_{A} D \lambda \text {. }
$$

Comparison of Eq. (67) with its solution kinetics analog given in Eq. (16) show striking similarities. However Eq. (67) is more complex than Eq. (16) owing to the contributions from the Bessel functions in $\lambda$ [Eq. (64)]. Expressions similar to Eq. (67) are often derived heuristically in textbooks. ${ }^{19}$ While such expressions are qualitatively correct they are quantitatively inaccurate. A careful treatment requires the application of Eq. (67).

We complete this section by finding the solution to Eq. (43) subject to the radiation boundary condition

$$
2 \pi R_{A} D\left(\frac{d W_{D 1}}{d r}\right)_{r=R_{A}}=k_{\theta q} W_{D 1}\left(R_{A}\right)
$$

in place of Eq. (45). The solution, found by elementary methods, is given by

$$
W_{D 1}(r)=J \tau\left[1+G K_{0}(\gamma r)+H I_{0}(\gamma r)\right],
$$

where

$$
\begin{aligned}
G= & -\left\{k_{\text {eq }} J \tau-\frac{C_{0} / J \tau-1}{I_{0}\left(\gamma R_{B}\right)}\left[2 \pi R_{A} D J \tau \gamma I_{1}\left(\gamma R_{A}\right)\right.\right. \\
& \left.-k_{\text {eq }} J \tau I_{0}\left(\gamma R_{A}\right)\right\} /\left\{2 \pi R_{A} D J \tau \gamma K_{1}\left(\gamma R_{A}\right)+k_{\text {eq }} J \tau K_{0}\left(\gamma R_{A}\right)\right. \\
& \left.+\frac{K_{0}\left(\gamma R_{B}\right)}{I_{0}\left(\gamma R_{B}\right)}\left[2 \pi R_{A} D J \tau \gamma I_{1}\left(\gamma R_{A}\right)-k_{\text {eq }} J \tau I_{0}\left(\gamma R_{A}\right)\right]\right\},
\end{aligned}
$$

and

$$
H=\frac{C_{0} / J \tau-1-G K_{0}\left(\gamma R_{B}\right)}{I_{0}\left(\gamma R_{B}\right)} .
$$

Using the properties of the Bessel functions we obtain for the infinite $R_{B}$ limit

$$
\begin{aligned}
\lim _{R_{B} \rightarrow \infty} W_{D 1}(r) & \equiv W_{D 1 \infty}(r), \\
& =J T\left[1-\frac{k_{\infty \phi} K_{0}(\gamma r)}{2 \pi R_{A} D y K_{1}\left(\gamma R_{A}\right)+k_{\infty Q} K_{0}\left(\gamma R_{A}\right)}\right] .
\end{aligned}
$$

The rate constant for the diffusion process is defined as usual to give

$$
\begin{aligned}
k & =\frac{2 \pi R_{A} D}{J \tau}\left(\frac{d W_{D \perp 0}}{d r}\right)_{r=R_{A}}, \\
& =2 \pi R_{A} D \frac{k_{00} \gamma K_{1}\left(\gamma R_{A}\right)}{2 \pi R_{A} D \gamma K_{1}\left(\gamma R_{A}\right)+k_{00} K_{0}\left(\gamma R_{A}\right)}, \\
& =\frac{k_{D} k_{00}}{k_{D}+k_{\text {eq }}},
\end{aligned}
$$

which is identical to the result given in Eqs. (21) and (34). As we indicated in Sec. II, Eq. (76) makes manifest that 


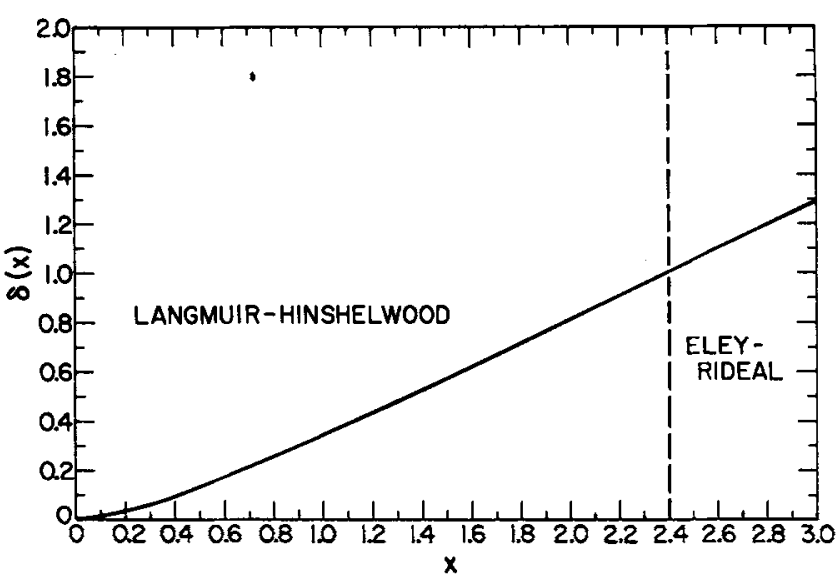

FIG. 2. Graph of the ratio of the Eley-Rideal rate to the Langmuir-Hinshelwood rate [Eq. (79)]. In most cases the Langmuir-Hinshelwood mechanism is dominant (i.e., $x \ll 2.4$ ). See the text.

$$
k_{D} \geq k
$$

and rate constants evaluated from Eq. (67) will give upper bounds to the true rate constants.

\section{Mechanistic considerations}

As mentioned in the Introduction, diffusion-influenced reactions by assumption must obey Langmuir-Hinshelwood kinetics as expressed by Eq. (1). We can address the importance of some Eley-Rideal mechanisms by examining Eq. (60). If we ignore those Eley-Rideal processes which occur via the physisorption of a precursor from the gas phase and only involve the direct interaction between gas phase and adsorbed reactants, then the Eley-Rideal rate is given by Eq. (61). We can then evaluate the ratio of the Eley-Rideal rate to the Langmuir-Hinshelwood rate from the defining relation

$$
\delta=\frac{F_{A}}{F_{D}} .
$$

Using Eqs. (61) and (63) we obtain

$$
\delta(x)=\frac{x K_{0}(x)}{2 K_{1}(x)}
$$

where

$$
x=\gamma R_{\mathrm{A}} \text {. }
$$

From the properties of Bessel functions we can evaluate the limits

$$
\lim _{x \rightarrow 0} \delta(x)=-\frac{x^{2} \ln x}{2}
$$

and

$$
\lim _{x \rightarrow \infty} \delta(x)=\frac{x}{2} .
$$

We see that the dominant mechanism is Langmuir-Hinshelwood for strongly bound reactants with large diffusion constants (small $x$ ). Conversely, the Eley-Rideal mechanism will be most important when the reactants are weakly bound with small diffusion constants (large $x$ ). A graph of $\delta(x)$ is given in Fig. 2. A rough mea- sure of the switch between the Eley-Rideal and Langmuir-Hinshelwood mechanisms occurs at the value of $x$-when $\delta(x)=1$. In Fig. 2 we have denoted the Langmuir-Hinshelwood and Eley-Rideal regions by dividing the graph at $x=2.4$ where $\delta(x)=1$.

To gain further insight into the relative importance of the two mechanisms in physical systems we note that for $R_{A}=2 \AA, x=2.4$ implies that $(D T)^{1 / 2} \cong 1 \AA$. Consequently, Langmuir-Hinshelwood behavior will occur when the average distance a reactant travels before desorption is on the order of a lattice spacing or greater. This is a very short distance, and we might expect most reactions to follow Langmuir-Hinshelwood kinetics. It is important to recognize that $\delta(x)$ is independent of $J$ so that the Langmuir-Hinshelwood process will predominate even if a large external flux of reactant molecules is applied to the surface. The Langmuir-Hinshelwood mechanism has been found experimentally to predominate for a variety of reactions. ${ }^{20,21}$ We see that an explanation for the dominance of Langmuir-Hinshelwood kinetics can be obtained from an analysis of Eq. (79).

\section{Behavior of the activation energy}

For diffusion-controlled surface reactions the activation energy is defined by

$$
E=-\frac{d \ln k_{\mathrm{p}}}{d \beta},
$$

where $\beta=1 / k_{B} T, k_{B}$ being the Boltzmann constant. If we assume both $D$ and $\tau$ to have Arrhenius behavior

$$
D=D_{0} \exp \left(-E_{D} \beta\right) \text {, }
$$

and

$$
\tau=\tau_{0} \exp \left(E_{A} \beta\right)
$$

and use Eq. (67) it is easy to show that

$$
\begin{aligned}
E & =E_{D}+\frac{\gamma}{k_{D}}\left(\frac{E_{A}-E_{D}}{2}\right) \frac{d k_{D}}{d \gamma}, \\
& =E_{D}+\frac{\gamma R_{A}\left(E_{A}-E_{D}\right)}{2}\left[\frac{K_{0}^{2}\left(\gamma R_{A}\right)+K_{1}^{2}\left(\gamma R_{A}\right)}{K_{0}\left(\gamma R_{A}\right) K_{1}\left(\gamma R_{A}\right)}\right] .
\end{aligned}
$$

For many systems we need to evaluate Eq. (87) for $\gamma R_{A}$ $\ll 1$ [See Sec. III C]. In this limit

$$
\lim _{\gamma \rightarrow 0} E=E_{D}+\frac{E_{D}-E_{A}}{2 \ln \left(R_{A} / \sqrt{D_{0} \tau_{0}}\right)+\beta\left(E_{D}-E_{A}\right)} .
$$

The small $\gamma$ approximation will be best at low temperatures by virtue of Eqs. (48), (84), and (85). From Eq. (88) we see that

$$
\lim _{\boldsymbol{T} \rightarrow 0} E=E_{D} \text {. }
$$

Consequently, for diffusion-controlled reactions the activation energy should approximately be equal to the activation energy for diffusion. From Eq. (88) we see that $E$ is a function of temperature. For surface reactions we can expect non-Arrhenius behavior at higher temperatures even for atom-atom recombination reactions where no steric effects are present. 
For diffusion-influenced reactions the experimental activation energy will exhibit the additional complications arising from the form of Eq. (76). When $k_{\mathrm{eq}}$ is very large relative to $k_{D}$ the reaction is diffusion controlled and the activation energy will be given by Eq. (87). When $k_{D}$ is large compared to $k_{\text {ee }}$ diffusion is unimportant, and the rate constant is approximately given by $k_{\text {eq }}$. For large $k_{D}$ the activation energy will be controlled by the activation energy associated with $k_{\text {eq }}$. To analyze the case when $k_{e q}$ and $k_{D}$ are comparable we write

$$
\begin{aligned}
& k_{D}=k_{D_{0}} \exp \left(-E_{D} \beta\right), \\
& k_{\text {eq }}=k_{\text {eq }} \exp \left(-E_{\text {eq }} \beta\right) .
\end{aligned}
$$

Using Eqs. (76) and (83) we obtain

$$
\begin{aligned}
E & =E_{D}+E_{e q}-\frac{E_{D} k_{D_{0}} \exp \left(-E_{D} \beta\right)+E_{\mathrm{eq}} k_{\mathrm{eq}} \exp \left(-E_{\mathrm{eq}} \beta\right)}{k_{D_{0}} \exp \left(-E_{D} \beta\right)+k_{\mathrm{eQ0}} \exp \left(-E_{\mathrm{eq}} \beta\right)} \\
& =\frac{k_{D} E_{\theta Q}+k_{\mathrm{eQ}} E_{D}}{k_{D}+k_{\mathrm{eq}}} .
\end{aligned}
$$

From Eq. (93) we can show that $E$ lies between $E_{D}$ and $E_{\text {eq }}$. For example, suppose $E_{\text {eq }}$ is greater than $E_{D}$. Using Eq. (93) we have

$$
E-E_{D}=\frac{k_{D}\left(E_{e q}-E_{D}\right)}{k_{D}+k_{\text {eq }}}>0
$$

and

$$
E-E_{e q}=\frac{k_{e Q}\left(E_{D}-E_{e Q}\right)}{k_{D}+k_{e q}}<0 .
$$

Consequently,

$$
E_{D} \leq E \leq E_{\text {eq }} \text {. }
$$

If $E_{\text {eq }}$ is less than $E_{D}$ we can similarly show that

$$
E_{\text {eq }} \leq E \leq E_{D} \text {. }
$$

From Eqs. (96) and (97) it is apparent that the activation energy for surface recombination processes is bounded by $E_{D}$ and $E_{0 q}$. In particular, the activation energy is not necessarily identical to the desorption energy.

\section{CONCLUSIONS}

Using ideas found to be successful in solution kinetics we have developed expressions to evaluate rate constants and activation energies for surface reactions. The expressions for diffusion-controlled rate constants are particularly useful for theoretical studies of surface reactions, since they provide an upper bound estimate of the true rate constant in terms of information about the diffusion constants for the reactive fragments. These fragment diffusion constants are determined by a portion of the entire potential energy surface that would be required for the complete treatment of reaction $\mathrm{dy}$ namics and are consequently more easily determined. As more complete potential surfaces become available the calculated rate constants can be refined by the applications of Eq. (76). We are using the formalism developed in this work to evaluate rate constants for heterogeneous atom-atom recombination reactions, and the results of these calculations will appear separately.

In solution kinetics rate constants for diffusion-controlled processes calculated from Eq. (16) are exact to the extent that the classical diffusion equation is exact. The corresponding approximations for diffusion-controlled surface reactions are more severe, because Eq. (42) is a two-dimensional representation of the true diffusion process. A more accurate approach to diffusion-controlled surface reactions would include surface binding forces and involve solutions to the corresponding Smoluchowski equation. We have avoided this approach, because the diffusion equation provides analytic expressions. We feel our solutions are at least qualitatively correct. Numerical studies to evaluate the errors in the two-dimensional model are in progress.

An approximation in the formalism presented in this work, which is less obvious than the imposition of twodimensional behavior, is the fact that the diffusion equation is known to be inaccurate at short distances from the absorber. ${ }^{8}$ To obtain accurate expressions it is necessary to solve the Fokker-Planck equation for the full phase-space distribution function, a difficult problem with absorbing boundary conditions. ${ }^{22}$ We are presently analyzing the errors in the diffusion equation by studying the equivalent Brownian dynamics problem.

\section{ACKNOWLEDGMENTS}

One of us (DLF) wishes to thank the Chemistry Division of Los Alamos National Laboratory for hospitality and support while on sabbatical leave from the University of Rhode Island. We wish to thank J. Winterkamp and Los Alamos National Laboratory's Group ESS-5 for computational support. We would also like to thank Francis Harlow for helpful discussions.

${ }^{1}$ S. H. Lin, K. P. Li, and H. Eyring, in Physical Chemistry: An Advanced Treatise, edited by H. Eyring (Academic, New York, 1975), Vol. 7, p. 1.

${ }^{2}$ J. D. Doll, and H. K. MeDowell, J. Chem. Phys. 77, 479 (1982).

${ }^{3}$ H. K. McDowell and J. D. Doll, Surf. Sci. 121, L537 (1982)

${ }^{4}$ J. D. Doll and H. K. McDowell, Surf. Sci. 129, 99 (1982).

${ }^{5} \mathrm{H}$. K. McDowell and J. D. Doll, J. Chem. Phys. (to be published).

${ }^{6}$ G. Ehrlich and K. Stolt, Annu. Rev. Phys. Chem. 31, 603 (1980).

${ }^{7}$ M. V. Smoluchowski, Z. Phys. Chem. 92, 129 (1917).

${ }^{8}$ S. Chandrasekhar, Rev. Mod. Phys. 15, 1 (1943).

${ }^{9}$ F. C. Collins and G. E. Kimball, J. Colloid Sci. 4, 425 (1949).

${ }^{10}$ M. J. Stowell, Philos. Mag. 26, 349 (1972).

${ }^{11} \mathrm{H}$. Wişe and B. J. Wood, Adv. At. Mol. Phys. 3, 291 (1967).

${ }^{12} \mathrm{P}$. W. Atkins, Physical Chemistry (Freeman, New York, 1978), Chap. 27.

${ }^{13}$ A. Szabo, D. Shoup. S. Northrup, and J. McCammon, J. Chem. Phys. 77, 4484 (1982).

${ }^{14} \mathrm{C}$. A. Emeis and P. L. Fehder, J. Am. Chem. Soc. 92, $2246(1970)$.

${ }^{15}$ M. N. Özisik, Boundary Value Problems of Heat Conduction 
(International Textbook, Scranton, 1968), Chap. 9.

${ }^{16} \mathrm{~A}$. Haji-Sheikh, Ph.D. thesis, University of Minnesota, 1965.

${ }^{17}$ See Ref. 12, Chap. 28.

${ }^{18} \mathrm{M}$. Abromowitz and I. A. Stegun, Handbook of Mathematical Functions (Natl. Bur. Stand. U.S. GPO, Washington, D.C., 1964), Chap. 9.
${ }^{19} \mathrm{G}$. A. Somorjal, Chemistry in Two Dimensions: Surfaces (Cornell University, Ithaca, 1981), Chap. 7.

${ }^{20}$ T. Engle and G. Ertl, Adv. Catal. 28, 1 (1979).

${ }^{21}$ C. T. Campbell, G. Ertl, H. Kuipers, and J. Segner, Surf. Sci. 107, 220 (1981).

${ }^{22}$ S. Harris, J. Chem. Phys. 75, 3103 (1981). 